\title{
Clinicopathological features and the impact of the new TNM classification of malignant tumors in patients with pulmonary large cell neuroendocrine carcinoma
}

\author{
AKIRA IYODA $^{1}$, SHI-XU JIANG ${ }^{2}$, WILLIAM D. TRAVIS ${ }^{3}$, NAOMI KUROUZU ${ }^{1}$, FUMIHIRO OGAWA ${ }^{1}$, \\ HIDEKI AMANO ${ }^{1}$, YUICHI SATO ${ }^{4}$, VALERIE W. RUSCH ${ }^{5}$, MAKOTO SAEGUSA $^{2}$ and YUKITOSHI SATOH ${ }^{1}$ \\ Departments of ${ }^{1}$ Thoracic Surgery and ${ }^{2}$ Pathology, Kitasato University, School of Medicine, Kanagawa 252-0374, Japan; \\ ${ }^{3}$ Department of Pathology, Thoracic Service, Memorial Sloan-Kettering Cancer Center, New York, NY 10065, USA; \\ ${ }^{4}$ Department of Applied Tumor Pathology, Graduate School of Medical Sciences, Kitasato University, \\ Kanagawa 252-0374, Japan; ${ }^{5}$ Department of Surgery, Thoracic Service, \\ Memorial Sloan-Kettering Cancer Center, New York, NY 10065, USA
}

Received July 12, 2012; Accepted February 1, 2013

DOI: $10.3892 /$ mco.2013.80

\begin{abstract}
The prognosis of patients with large-cell neuroendocrine carcinoma (LCNEC) of the lung is extremely poor and the optimal treatment for these patients has yet to be determined. In this study, we described the clinicopathological characteristics of LCNECs and compared the prognoses of corresponding stages determined by the guidelines of the 6th and 7th editions of the TNM classification of malignant tumors. Clinical data from 42 patients diagnosed with primary LCNEC who underwent treatment at Kitasato University Hospital between 1991 and 2009 were retrospectively analyzed. On follow-up of 42 patients, 22 (52.4\%) had confirmed recurrent tumors, including 8 patients with mediastinal lymph node recurrences and 19 with distant metastases. The sites of distant metastases included the brain in 8 , bone in 8 , liver in 7 , lungs in 5 and adrenal glands in 4 patients. For all the patients, the 5-year overall survival rate was $34.7 \%$ and the 5 -year disease-free survival rate was $32.9 \%$. The 5 -year overall survival rates of patients with stage I cancers according to the 6 th and 7 th staging editions was $51.3 \%$ (6th $\mathrm{n}=18,7 \mathrm{th} \mathrm{n}=16$ ). Thirteen of 42 patients $(31.0 \%)$ also had metachronous or synchronous primary cancers. Patients with LCNEC had poor outcomes, even those with stage I tumors classified according to the 7th edition of the TNM classification. Therefore, frequent recurrences in addition to metachronous or synchronous primary cancers in patients with LCNEC should be treated.
\end{abstract}

Correspondence to: Dr Akira Iyoda, Department of Thoracic Surgery, Kitasato University, School of Medicine, 1-15-1 Kitasato, Sagamihara, Kanagawa 252-0374, Japan

E-mail: aiyoda@med.kitasato-u.ac.jp

Key words: large cell, neuroendocrine, TNM stage, prognosis, outcome

\section{Introduction}

Large-cell neuroendocrine carcinomas (LCNECs) of the lung are aggressive tumors. Patients with LCNEC have an extremely poor prognosis since the biological behavior of LCNECs is similar to that of small cell lung carcinomas and LCNECs also have characteristics of high-grade neuroendocrine tumors (1-9). However, the treatment for patients with LCNEC has been based on non-small cell carcinomas. To improve the outcomes for patients with LCNEC, a better understanding of its clinicopathological characteristics, including preoperative diagnoses, the effectiveness of adjuvant chemotherapy, tumor recurrence rates and the prognosis of different stages, is required. In addition, a new edition (7th) of the TNM classification of malignant tumors was introduced in 2007 by the International Association for the Study of Lung Cancer (IASLC) to replace the 6th edition (10). At present, the prognosis of LCNEC tumors staged according to the newer edition of the TNM classification is unknown. The aim of this study was to describe the clinicopathological features of LCNECs in detail and compare outcomes between stages determined by the guidelines of the 6th and 7th editions. We also discuss the role of adjuvant chemotherapy and the optimal management of patients with LCNEC.

\section{Patients and methods}

Ethics. The Institutional Review Board of Kitasato University Hospital approved the protocols and procedures used in the study. As this was a retrospective study, treatments varied, particularly adjuvant chemotherapies.

Patients. Clinical data from 42 patients diagnosed with primary LCNEC who underwent treatment at the Kitasato University Hospital between 1991 and 2009 were retrospectively analyzed. LCNEC was diagnosed in resected surgical specimens showing evidence of neuroendocrine 
differentiation detected by immunohistochemistry and neuroendocrine morphologic features according to the World Health Organization International Histological Classification of Tumors (1).

Immunohistochemical staining used the following antibodies: polyclonal anti-chromogranin A (Dako, Glostrup, Denmark), polyclonal antisynaptophysin (Dako) and antiNCAM (Nippon Kayaku Co., Tokyo, Japan). Positive staining for chromogranin A, synaptophysin or NCAM indicated neuroendocrine differentiation $(1,11)$.

The following data were gathered from the medical records: patient gender, age, smoking index, clinical staging, preoperative symptoms, presence of paraneoplastic syndrome, tumor sites, definitive preoperative diagnosis, preoperative serum tumor marker levels [carcinoembryonic antigen (CEA), squamous cell carcinoma antigen (SCC-A), neuron-specific enolase (NSE), cytokeratin 19 fragment (CYFRA) and progastrin- releasing peptide (ProGRP)], surgical procedure, pathological findings (tumor size, mitotic rate, surgical margin, immunohistochemical findings), pathological TNM stages according to the 6th and 7th editions, adjuvant chemotherapy, time of recurrence, time of mortality, date of last follow-up, recurrence site(s), occurrence of metachronous or synchronous primary cancer and patient outcome.

Treatments and evaluation. Preoperative chest computed tomography (CT) was performed to evaluate the primary tumor, lymph node metastases, pulmonary metastases and pleural effusion. To evaluate distant metastases, magnetic resonance imaging (MRI) or CT were used for brain lesions, abdominal CT for liver and adrenal gland metastases, bone scintigraphy for bone metastases and in certain cases, positron emission tomography was used for distant metastases.

The response of LCNEC tumors to induction chemotherapy was based on the Response Evaluation Criteria in Solid Tumors (RECIST) Guidelines (12). Postoperative follow-ups of patients with LCNEC were routinely performed 3 or 4 times per year and consisted of screening for symptoms and chest roentgenography. When abnormal findings were identifed, the patient underwent chest $\mathrm{CT}$ and if recurrences were suspected, the patient also underwent abdominal CT, brain MRI or CT, bone scintigraphy or positron emission tomography.

Statistical analysis. Survival time was calculated from the date of surgery to the time of recurrence or mortality or date of last follow-up and was evaluated using the Kaplan-Meier method. Survival curves were compared using the log-rank test. $\mathrm{P}<0.05$ was considered to indicate a statistically significant difference.

\section{Results}

Patient characteristics. The 42 patients with LCNEC were predominantly male, predominantly smokers and none of the patients manifested paraneoplastic syndrome (Table I). With regard to preoperative serum tumor markers, CEA was elevated in $50 \%$ of patients, whereas NSE and ProGRP, specific neuroendocrine tumor markers, were infrequently
Table I. Preoperative characteristics of 42 patients with pulmonary large-cell neuroendocrine carcinoma (LCNEC).

\begin{tabular}{lc}
\hline Variable & $\mathrm{N}(\%)$ \\
\hline Total & $42(100)$ \\
Age (years) & \\
Mean (range) \pm SD & $64.4(50-85) \pm 8.4$ \\
Gender & \\
Male & $38(90.5)$ \\
Female & $4(9.5)$ \\
Clinical stage & \\
IA & $11(26.2)$ \\
IB & $13(31.0)$ \\
IIA & $1(2.4)$ \\
IIB & $8(19.0)$ \\
IIIA & $8(19.0)$ \\
IIIB & $0(0.0)$ \\
IV & $1(2.4)$ \\
Smoking & \\
Smoker & \\
Non-smoker & $40(95.2)$ \\
Symptomatic & $2(4.8)$ \\
+ &
\end{tabular}

SD, standard deviation.

elevated (Table II). There were 6 patients whose preoperative diagnoses were definitive or suspicious for LCNEC. Limited resection was performed in only 2 cases and intraoperative pleural lavage cytology was positive in only 1 case (Table III).

Pathological findings. Pathological findings revealed that 37 patients underwent complete resection, with negative tumor margins. There were 11 patients whose tumors were diagnosed as combined LCNEC with components of adenocarcinoma in 7, squamous cell carcinoma in 3 and large cell carcinoma in 1 case. The mean number of mitoses per 10 high-power fields was 63.7 (Tables IV and V). Immunohistochemical staining revealed that no neuroendocrine marker was expressed in every LCNEC case and there were 12 cases that were only positive for 1 marker (Table IV). 
Table II. Preoperative serum tumor markers.

\begin{tabular}{lr}
\hline Tumor marker & $\mathrm{N}(\%)$ \\
\hline CEA (38 informative cases) & $19(50.0)$ \\
Elevated & $19(50.0)$ \\
Not & \\
CYFRA (18 informative cases) & $5(27.8)$ \\
Elevated & $13(72.2)$ \\
Not & \\
NSE (35 informative cases) & $9(25.7)$ \\
Elevated & $26(74.3)$ \\
Not & $4(19.0)$ \\
ProGRP (21 informative cases) & $17(81.0)$ \\
Elevated & $4(11.8)$ \\
Not & $30(88.2)$ \\
SCC-A (34 informative cases) & \\
Elevated & \\
Not & \\
\hline CEA, carcinoembryonic antigen; CYFRA, cytokeratin & \\
NSE, neuron-specific enolase; ProGRP, progastrin-releasing peptide; \\
SCC-A, squamous cell carcinoma antigen. \\
\end{tabular}

Table III. Surgical procedures.

\begin{tabular}{lc}
\hline Variable & $\mathrm{N}(\%)$ \\
\hline Total & $42(100.0)$ \\
Surgery & $5(11.9)$ \\
Pneumonectomy & $3(7.1)$ \\
Bilobectomy & $32(76.2)$ \\
Lobectomy & $2(4.8)$ \\
Wedge resection & \\
Plasty & $1(2.4)$ \\
Pulmonary artery & $1(2.4)$ \\
Carina & \\
Intraoperative pleural & \\
lavage cytology & \\
(26 informative cases) & \\
Positive & $1(3.8)$ \\
Negative & $25(96.2)$ \\
Combined resection & \\
Chest wall & $3(7.1)$ \\
Parietal pleura & $2(4.8)$ \\
Carina + esophagus & $1(2.4)$ \\
Pericardium & $1(2.4)$ \\
\hline
\end{tabular}

TNM classifications according to the 6th and 7th editions and prognosis. Comparison of the pathological classifications of the 7th and 6th edition of the TNM classification revealed that in the newer classification, 6 cases were reclassified from
Table IV. Postoperative characteristics of 42 patients with pulmonary large-cell neuroendocrine carcinoma (LCNEC).

\begin{tabular}{|c|c|}
\hline Variable & $\mathrm{N}(\%)$ \\
\hline Total & $42(100.0)$ \\
\hline \multicolumn{2}{|l|}{ Surgical margin } \\
\hline Negative & $37(88.1)$ \\
\hline Microscopic-positive & $4(9.5)$ \\
\hline Macroscopic-positive & $1(2.4)$ \\
\hline \multicolumn{2}{|l|}{ Pure or combined } \\
\hline LCNEC & $31(73.8)$ \\
\hline Combined LCNEC & $11(26.2)$ \\
\hline Adenocarcinoma & 7 \\
\hline Squamous cell ca & 3 \\
\hline Large cell ca & 1 \\
\hline \multicolumn{2}{|l|}{ Tumor size (cm) } \\
\hline Mean (range) $\pm \mathrm{SD}$ & $4.0(1.4-9.0) \pm 1.8$ \\
\hline \multicolumn{2}{|l|}{ Mitoses/10 hpf } \\
\hline Mean (range) $\pm \mathrm{SD}$ & $63.7(16-141) \pm 30.7$ \\
\hline \multicolumn{2}{|l|}{$\begin{array}{l}\text { Neuroendocrine marker } \\
\text { Chromogranin }\end{array}$} \\
\hline Positive & $21(50.0)$ \\
\hline Negative & $21(50.0)$ \\
\hline \multicolumn{2}{|l|}{ Synaptophysin } \\
\hline Positive & $35(83.3)$ \\
\hline Negative & $7(16.7)$ \\
\hline \multicolumn{2}{|l|}{ NCAM } \\
\hline Positive & $34(81.0)$ \\
\hline Negative & $8(19.0)$ \\
\hline \multicolumn{2}{|c|}{ Number of positive markers } \\
\hline 1 & $12(28.6)$ \\
\hline 2 & $12(28.6)$ \\
\hline All 3 & $18(42.9)$ \\
\hline
\end{tabular}

Ca, carcinoma; SD, standard deviation; hpf, high-power field.

stage IIIB of the older classification to stage IIB or IIIA, 4 from stage IA to stage IB, 3 from stage IIB to stage IIA or IIIA and 2 from stage IB to stage IIA. For the patients, the 5-year overall survival rate was $34.7 \%$ and the 5-year disease-free survival rate was $32.9 \%$. The 5-year overall survival rates of patients with stage I cancers according to the 6th and 7th staging editions was $51.3 \%$. In the two classifications, patients with LCNEC had extremely poor outcomes, even for stage I cancers (Table VI and Fig. 1). The 3-year overall survival rate of patients with pure LCNEC was $38.5 \%$ and that of patients with combined LCNEC was $17.9 \%$. There was no significant difference between the survival rates of pure LCNEC and combined LCNEC $(\mathrm{P}=0.7546)$.

Induction and adjuvant therapy. Five patients underwent platinum-based induction chemotherapy and 3 patients achieved partial responses (response rate, 60\%). Nine patients under- 
A

C
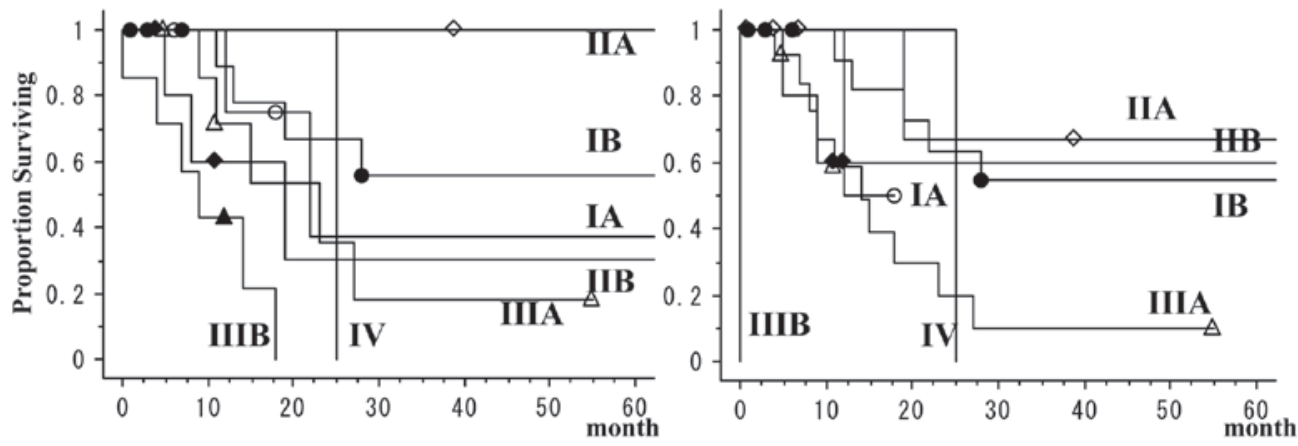

B

D
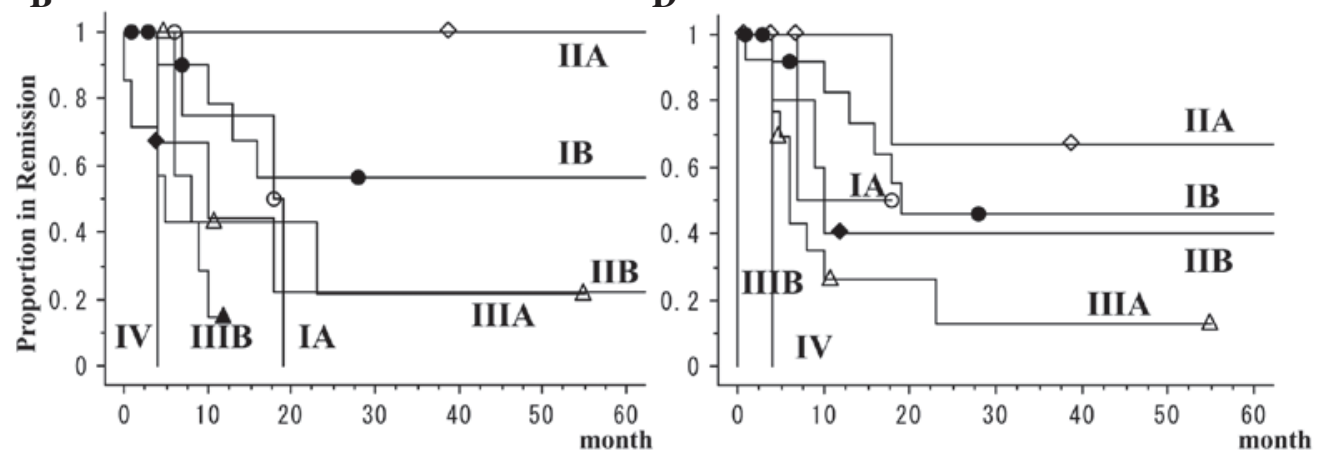

Figure 1. (A) Overall survival curves of patients with large cell neuroendocrine carcinoma (LCNEC) according to the 6th edition of the TNM classification of malignant tumors. (B) Disease-free survival curves of patients with LCNEC according to the 6th edition of the TNM classification of malignant tumors. (C) Overall survival curves of patients with LCNEC according to the 7th edition of the TNM classification of malignant tumors. (D) Disease-free survival curves of patients with LCNEC according to the 7th edition of the TNM classification of malignant tumors.

Table V. Mitoses in 40 patients with large-cell neuroendocrine carcinoma.

Mitoses/10 hpf

No. of cases

11 to 20

21 to 30 3

31 to 40 1

41 to 50

51 to 60

61 to 70

71 to 80

81 to 90

91 to 100

$>100$

Hpf, high-power field.

went adjuvant chemotherapy, consisting of platinum-based chemotherapy in 5 and non-platinum-based chemotherapy in 4 cases (Table VII). Three of the latter patients, who were staged IB and IIB according to the 6th edition, received uracil and tegafur (UFT).

Recurrences and other cancers. Postoperative recurrences were observed in 22 cases $(52.4 \%)$. Recurrences consisted of local mediastinal lymph node recurrences in 3 , mediastinal lymph node recurrences with distant metastases in 5, localized recurrence in the diaphragm with distant metastases in 1 and distant metastases only in 13 patients (Table VII). Distant metastases were frequently observed in the brain, bone, liver, lung and adrenal glands (Table VIII).

Of the 37 patients with complete tumor resection, $18(48.6 \%)$ had postoperative recurrences and 16 of those had distant metastases. In 15 patients with pathological stage I tumors according to the 7th edition of the TNM classification, $7(46.7 \%)$ had distant metastases without local recurrences. In 26 patients undergoing surgery alone without induction or adjuvant therapy, $13(50.0 \%)$ had postoperative recurrences, of which 12 had distant metastases. Of the 9 patients undergoing adjuvant chemotherapy, only 2 who had undergone UFT adjuvant chemotherapy had distant metastases. Four of 5 patients undergoing platinum-based adjuvant chemotherapy had no recurrences. Of the 22 patients with postoperative recurrences, 21 developed recurrences $<2$ years after surgery (Table IX).

Of 42 patients, $13(31.0 \%)$ had metachronous or synchronous primary cancers and 3 patients had primary cancers at $>1$ site with the exception of LCNEC tumors (Table X). Of the 13 cases, only 3 cases had undergone induction or adjuvant chemotherapy.

\section{Discussion}

In 2007, IASLC published a new staging system; however, no studies are available on the correlations between the new staging system and the prognoses of LCNEC stages. In the 
Table VI. Pathological staging according to the 6th and 7th editions of the TNM classification.

\begin{tabular}{|c|c|c|c|}
\hline Variable & $\mathrm{N}(\%)$ & 5 -year overall survival rate $(\%)$ & 5 -year disease-free survival rate $(\%)$ \\
\hline \multicolumn{4}{|c|}{ 6th edition pathological stage } \\
\hline IA & $6(14.3)$ & 37.5 & 0 \\
\hline IB & $12(28.6)$ & 55.6 & 56.3 \\
\hline IIA & $2(4.8)$ & 100 & 100 \\
\hline IIB & $6(14.3)$ & 30.0 & 22.2 \\
\hline IIIA & $8(19.0)$ & - & - \\
\hline IIIB & $7(16.7)$ & 0 & - \\
\hline IV & $1(2.4)$ & 0 & 0 \\
\hline \multicolumn{4}{|c|}{ 7th edition pathological stage } \\
\hline IA & $2(4.8)$ & - & - \\
\hline IB & $14(33.3)$ & 54.5 & 45.8 \\
\hline IIA & $6(14.3)$ & 66.7 & 66.7 \\
\hline IIB & $5(11.9)$ & 60.0 & 40.0 \\
\hline IIIA & $13(31.0)$ & - & - \\
\hline IIIB & $1(2.4)$ & 0 & 0 \\
\hline IV & $1(2.4)$ & 0 & 0 \\
\hline
\end{tabular}

Table VII. Adjuvant therapies and recurrence.

\begin{tabular}{lc}
\hline Variable & $\mathrm{N}(\%)$ \\
\hline Total & $42(100.0)$ \\
Therapy & \\
Induction therapy & $5(11.9)$ \\
Chemotherapy & $14(33.3)$ \\
Adjuvant therapy & $9(21.4)$ \\
Chemotherapy & $5(11.9)$ \\
Platinum-based & $4(9.5)$ \\
Non-platinum-based & $5(11.9)$ \\
Radiation therapy & \\
Postoperative recurrence & $22(52.4)$ \\
Positive & $20(47.6)$ \\
Negative & \\
Site of recurrence & $3(7.1)$ \\
Local & $6(14.3)$ \\
Local + distant & $13(31.0)$ \\
Distant &
\end{tabular}

present study, we have demonstrated that LCNEC patients had extremely poor outcomes, even for stage I disease according to the new staging system. Studies published prior to 2007 have also reported poor outcomes for patients with LCNEC, with 5-year survival rates ranging from 15 to $57 \%$ (2-4,13-19). Even LCNEC patients at pathological stage I had poor outcomes, with 5-year survival rates of 27-67\%. Therefore, according to the new TNM staging system, the prognoses of patients with different stages of LCNEC have not changed.

In patients with LCNEC who have had surgery as initial treatment, knowledge of the frequent sites of recurrence is
Table VIII. Distant metastases in patients with large-cell neuroendocrine carcinoma.

\begin{tabular}{lc}
\hline Site & No. of cases \\
\hline Brain & 8 \\
Bone & 8 \\
Live & 7 \\
Lung & 5 \\
Adrenal gland & 4 \\
\hline & \\
& \\
Table IX. Duration from date of surgery until tumor recurrence. & \\
\hline Duration (months) & No. of cases \\
\hline$\leq 3$ & 1 \\
$4-6$ & 9 \\
$7-12$ & 5 \\
$13-24$ & 6 \\
$25-36$ & 0 \\
$\geq 37$ & 1 \\
\hline
\end{tabular}

required for treatment planning. In this study, we revealed that 21 of 22 patients with recurrent tumors developed their recurrences within 2 years after surgery. There has been limited information published on treatments for patients with LCNEC, particularly with regard to recurrent LCNEC tumors and sites of recurrence. In a study of LCNEC patients who were followed over time, the majority of recurrent tumors occurred within 3 years after surgery; patients frequently developed brain metastases and a number of patients with recurrence had extremely poor 
Table X. Metachronous or synchronous primary cancers in patients with large cell neuroendocrine carcinoma.

\begin{tabular}{ll} 
Metachronous or synchronous primary cancers & N \\
\hline Lung ca & 3 \\
Gastric ca & 2 \\
Esophageal ca & 1 \\
Ca of the uterine cervix & 1 \\
Ca of the uterine body & 1 \\
Colon ca & 1 \\
Urinary bladder ca & 1 \\
Pharyngeal ca + lung ca & 1 \\
Colon ca + lung ca & 1 \\
Esophageal ca + prostate ca & 1
\end{tabular}

$\mathrm{Ca}$, carcinoma

outcomes. However, certain cases with recurrence achieved good responses to treatment, particularly treatment with platinum-based chemotherapy, radiation therapy or a combination thereof (20). Findings of another study demonstrated that LCNEC tumors responded well to chemotherapy (21). These results indicate that it is necessary to treat recurrences as early as possible after the initial surgery and recurrent tumors should be treated using platinum-based chemotherapy, radiation therapy or chemoradiotherapy with platinum-based agents.

As a number of patients with recurrent LCNEC tumors had an extremely poor outcome, preventing recurrent tumors is crucial. Promising results of previous studies indicate that the efficacy of adjuvant chemotherapy for patients with LCNEC should be examined $(15,22,23)$. In the prospective study initiated in 2000 by Iyoda et al (23), treatment using cisplatin plus etoposide was evaluated, since it is similar to therapy used for small cell lung carcinoma, which has clinicopathological and biological features resembling LCNEC. The results revealed that patients undergoing cisplatin-plus-etoposide-based adjuvant chemotherapy after complete surgical resection achieved good outcomes. In their retrospective study, Rossi et al (15) also reported that cisplatin-plus-etoposide-based adjuvant chemotherapy was effective for patients with LCNEC. Thus, adjuvant chemotherapy following complete resection of LCNEC tumors appears promising for achieving good outcomes. Moreover, platinum-based adjuvant chemotherapy for LCNEC patients following surgery significantly prevented recurrence (20). Multivariate analyses revealed that platinum-based adjuvant chemotherapy was a significant, good prognostic factor in patients with LCNEC, although propensity score analyses did not confirm this observation. In the present study, platinum-based adjuvant chemotherapy appeared to protect patients from recurrences.

Although it is important to check for LCNEC tumor recurrence, we must also be aware of additional new cancer lesions, including those at other sites. The incidence of a second primary digestive cancer following resection of lung cancer has been reported to be $1-2 \%$ (24). The proportion of patients successfully treated for their initial non-small cell lung carcinoma and at risk of developing a second non-small cell lung carcinoma has been reported to be $1-2 \%(25,26)$. A previous study has also revealed a high rate of postoperative secondary cancers following surgery for LCNEC tumors (20). Our results demonstrated that $31 \%$ of LCNEC patients had metachronous or synchronous primary cancer. These results indicate that a high proportion of LCNEC patients develop a second primary cancer. Although LCNEC tumors are intrinsically aggressive, development of second primary cancers may be one of the reasons that patients with LCNEC have poor outcomes. We need to consider the risk of the occurrence of second primary cancers when following postoperative LCNEC patients and continue to investigate the mechanisms involved in the frequent development of metachronous or synchronous primary cancers in these patients.

In conclusion, our results have shown that the histological classifications of LCNEC were reproducible and patients with LCNEC had poor outcomes, even for stage I disease in the new TNM staging system. Frequent recurrences and metachronous or synchronous primary cancers in patients with LCNEC should be treated. Adjuvant chemotherapy with platinum-based regimens may be effective in preventing recurrent tumors.

\section{Acknowledgements}

The authors thank Isao Okayasu, Noriyuki Masuda, Kazushige Hayakawa, Hidenori Hara, Yoshio Matsui and Kenji Nezu for their helpful and excellent contributions. This study was supported in part by a Grant-in-aid for Scientific Research (C) 24592098 of the Japanese Ministry of Education, Culture, Sports, Science and Technology.

\section{References}

1. Travis WD, Colby TV, Corrin B, Shimosato Y and Brambilla E (eds): Histological Typing of Lung and Pleural Tumours. World Health Organization International Histological Classification of Tumors, XIII. 3rd edition. Springer-Verlag, Berlin/Heidelberg, 1999.

2. Iyoda A, Hiroshima K, Nakatani Y and Fujisawa T: Pulmonary large cell neuroendocrine carcinoma: its place in the spectrum of pulmonary carcinoma. Ann Thorac Surg 84: 702-707, 2007.

3. Iyoda A, Hiroshima K, Toyozaki T, Haga Y, Fujisawa T and Ohwada H: Clinical characterization of pulmonary large cell neuroendocrine carcinoma and large cell carcinoma with neuroendocrine morphology. Cancer 91: 1992-2000, 2001.

4. Asamura H, Kameya T, Matsuno Y, et al: Neuroendocrine neoplasms of the lung: a prognostic spectrum. J Clin Oncol 24: 70-76, 2006.

5. Iyoda A, Hiroshima K, Moriya Y, et al: Pulmonary large cell neuroendocrine carcinoma demonstrates high proliferative activity. Ann Thorac Surg 77: 1891-1895, 2004.

6. Iyoda A, Hiroshima K, Baba M, Saitoh Y, Ohwada H and Fujisawa T: Pulmonary large cell carcinomas with neuroendocrine features are high-grade neuroendocrine tumors. Ann Thorac Surg 73: 1049-1054, 2002.

7. Onuki N, Wistuba II, Travis WD, et al: Genetic changes in the spectrum of neuroendocrine lung tumors. Cancer 85: 600-607, 1999.

8. Jones MH, Virtanen C, Honjoh D, et al: Two prognostically significant subtypes of high-grade lung neuroendocrine tumours independent of small-cell and large-cell neuroendocrine carcinomas identified by gene expression profiles. Lancet 363: 775-781, 2004.

9. Hiroshima K, Iyoda A, Shibuya K, et al: Genetic alterations in early-stage pulmonary large cell neuroendocrine carcinoma. Cancer 100: 1190-1198, 2004. 
10. Goldstraw P, Crowley J, Chansky K, et al: International Association for the Study of Lung Cancer International Staging Committee. The IASLC Lung Cancer Staging Project: proposals for the revision of the TNM stage groupings in the forthcoming (seventh) edition of the TNM Classification of malignant tumours. J Thorac Oncol 2: 706-714, 2007.

11. Jiang SX, Kameya T, Shoji M, Dobashi Y, Shinada J and Yoshimura H: Large cell neuroendocrine carcinoma of the lung. A histologic and immunohistochemical study of 22 cases. Am J Surg Pathol 22: 526-537, 1998.

12. Therasse P, Arbuck SG, Eisenhauer EA, et al: New guidelines to evaluate the response to treatment in solid tumors. European Organization for Research and Treatment of Cancer, National Cancer Institute of the United States, National Cancer Institute of Canada. J Natl Cancer Inst 92: 205-216, 2000.

13. Veronesi G, Morandi U, Alloisio M, et al: Large cell neuroendocrine carcinoma of the lung: A retrospective analysis of 144 surgical cases. Lung Cancer 53: 111-115, 2006.

14. Takei H, Asamura H, Maeshima A, et al: Large cell neuroendocrine carcinoma of the lung: a clinicopathologic study of eighty-seven cases. J Thorac Cardiovasc Surg 124: 285-292, 2002 .

15. Rossi G, Cavazza A, Marchioni A, et al: Role of chemotherapy and the receptor tyrosine kinases KIT, PDGFRalpha, PDGFRbeta, and Met in large-cell neuroendocrine carcinoma of the lung. J Clin Oncol 23: 8774-8785, 2005.

16. Travis WD, Rush W, Flieder DB, et al: Survival analysis of 200 pulmonary neuroendocrine tumors with clarification of criteria for atypical carcinoid and its separation from typical carcinoid. Am J Surg Pathol 22: 934-944, 1998.

17. Paci M, Cavazza A, Annessi V, et al: Large cell neuroendocrine carcinoma of the lung: a 10-year clinicopathologic retrospective study. Ann Thorac Surg 77: 1163-1167, 2004.
18. Battafarano RJ, Fernandez FG, Ritter J, et al: Large cell neuroendocrine carcinoma: an aggressive form of non-small cell lung cancer. J Thorac Cardiovasc Surg 130: 166-172, 2005.

19. Skuladottir H, Hirsch FR, Hansen HH and Olsen JH: Pulmonary neuroendocrine tumors: incidence and prognosis of histological subtypes. A population-based study in Denmark. Lung Cancer 37: 127-135, 2002

20. Iyoda A, Hiroshima K, Moriya Y, et al: Postoperative recurrence and the role of adjuvant chemotherapy in patients with pulmonary large-cell neuroendocrine carcinoma. J Thorac Cardiovasc Surg 138: 446-453, 2009.

21. Yamazaki S, Sekine I, Matsuno Y, et al: Clinical responses of large cell neuroendocrine carcinoma of the lung to cisplatin-based chemotherapy. Lung Cancer 49: 217-223, 2005.

22. Iyoda A, Hiroshima K, Toyozaki T, et al: Adjuvant chemotherapy for large cell carcinoma with neuroendocrine features. Cancer 92: 1108-1112, 2001.

23. Iyoda A, Hiroshima K, Moriya Y, et al: Prospective study of adjuvant chemotherapy for pulmonary large cell neuroendocrine carcinoma. Ann Thorac Surg 82: 1802-1807, 2006.

24. Kamiyama H, Ikeya T, Suda K, Murai K, Aoyama K and Hoshi E: Second primary digestive cancer after resection of lung cancer. Surg Today 34: 577-580, 2004

25. Battafarano RJ, Force SD, Meyers BF, et al: Benefits of resection for metachronous lung cancer. J Thorac Cardiovasc Surg 127: 836-842, 2004.

26. Johnson BE: Second lung cancers in patients after treatment for an initial lung cancer. J Natl Cancer Inst 90: 1335-1345, 1998. 